Research report

\title{
Illusory self-identification with an avatar reduces arousal responses to painful stimuli
}

\author{
Daniele Romano $^{\mathrm{a}, \mathrm{b}, \mathrm{c}}$, Christian Pfeiffer ${ }^{\mathrm{b}, \mathrm{c}}$, Angelo Maravita ${ }^{\mathrm{a}}$, Olaf Blanke ${ }^{\mathrm{b}, \mathrm{c}, *}$ \\ a Università degli studi di Milano Bicocca, Department of Psychology, Piazza Ateneo Nuovo 1, Milano 20126, Italy \\ b Center for Neuroprosthethics, School of Life Sciences, Ecole Polytechnique Fédérale de Lausanne, Station 19, Lausanne CH-1015, Switzerland \\ ${ }^{\mathrm{c}}$ Laboratory of Cognitive Neuroscience, Brain Mind Institute, School of Life Sciences, Ecole Polytechnique Fédérale de Lausanne, Station 19, Lausanne \\ $\mathrm{CH}-1015$, Switzerland
}

\section{H I G H L I G H T S}

- We experimentally induced the full body illusion through a robotic device.

- We collected SCRs and ratings following acute pain stimulation during bodily illusion.

- Reduced arousal responses were detectable under illusory states of self-consciousness.

- Reduced SCR was related to the degree of ownership experienced for the virtual body.

- Virtual body must be in anatomical configuration to be effective.

\section{A R T I C L E I N F O}

\section{Article history:}

Received 2 October 2013

Received in revised form

11 December 2013

Accepted 21 December 2013

Available online 8 January 2014

\section{Keywords:}

Full body illusion

Pain

Analgesia

Body representation

Self-identification

Skin conductance response

\begin{abstract}
A B S T R A C T
Looking at one's own body has been shown to induce analgesia. In the present work we investigated whether illusory self-identification with an avatar, as induced experimentally through visuo-tactile stimulation, modulates the response to painful stimuli.

In 30 healthy volunteers, a robotic device was used to stroke the participants' back, while they viewed either the body of an avatar, a non-body object (control object), or a body avatar with scrambled body parts (control body). All were visually stimulated in either congruent or incongruent fashion with the participant's body. We collected physiological responses (skin conductance response: SCR) to painful stimuli delivered to the participant's hand and responses to a questionnaire inquiring about self-identification with the avatar. We expected reduced physiological responses to pain during the observation of a body avatar only during synchronous visuo-tactile stroking and no reduction for the control object and the control body.

Results showed a reduced SCR to painful stimuli when participants observed the normal body avatar being stroked synchronously that was also associated with largest self-identification ratings recordable already during the pain anticipation. Moreover, a negative correlation between self-identification and SCR was observed, suggesting that a greater degree of self-identification with the avatar was associated with larger decreases in SCR. These results suggest that during states of illusory self-identification with the avatar, the vision of an alien body (anatomically compatible for the vision and congruently stroked for the touch) is effective in modulating physiological responses to painful stimuli.
\end{abstract}

(c) 2014 Elsevier B.V. All rights reserved.

\section{Introduction}

Bodily self-consciousness is not considered anymore a unitary inviolable concept. Recent experimental evidences suggest that it

\footnotetext{
* Corresponding author at: Corresponding author. Tel.: +4121 6931822; fax: +41216931770.

E-mail addresses: d.romano10@campus.unimib.it (D. Romano), christian.pfeiffer@epfl.ch (C. Pfeiffer), angelo.maravita@unimib.it (A. Maravita), olaf.blanke@epfl.ch (O. Blanke).
}

is rather a result of multisensory bodily signal integration in the brain. Bodily self-consciousness has been proposed to comprise self-identification (the experience that 'I' identify with a body), selflocation (the experience of where 'I' am located), and a first-person perspective (from where 'I' experience the world), but also relating to the sense of agency (the experience that 'I' am the agent causing 'my' actions) [1-3]. Since the first experimental induction of changes in limb-ownership and location in the rubber-hand illusion [4], further studies have demonstrated that it is possible to extend one's own body representation to different external objects such as a prosthetic hand [4] or different fake body parts [5-8] but 
also to entire bodies $[9,10]$. In the rubber hand illusion (RHI), the visuo-tactile congruent stimulation of one's own hidden hand and a visible anatomical compatible fake hand induces the sensation that the prosthetic limb belongs to oneself [4,11]. Similarly, the full body illusion (FBI) can be induced; thus, congruent visuo-tactile stimulation at the trunk can induce self-identification and self-location changes with respect to a virtual or fake body $[12,13,9,14]$.

Multisensory body representation has been proposed to be crucial for self-identification with the body and for other aspects of bodily self-consciousness $[4,15,9,16,17,11]$; however, it has also been shown to be critical for any sensory perception including pain.

Recent studies from cognitive neuroscience show that although pain is highly subjective, it is affected by certain bodily states and experimentally modulated multisensory signals [18-20]. Thus, although nociceptive stimuli are processed through specific sensory pathways [18,21], similar to non painful stimuli, pain can be critically modulated by vision.

Previous work has shown that looking at one's own body but not to an object or at another person's body, while receiving a painful stimulus, produces analgesic effects [22,20]. Starting from this observation, we aimed at investigating the relationship between pain processing and body ownership; here, we sought for evidence that reduced responses to nociceptive stimuli can be obtained not only by looking at one's own body [20] but also when looking at another person's body or avatar, especially under conditions of self-identification with the virtual body. Thus, we asked whether changes in illusory self-identification following the induction of an FBI would be associated with a reduction of pain responses.

In two experiments, we combined robotic stimulation and virtual reality technology in order to induce the FBI [23-25]. We then investigated the response to acute noxious stimuli delivered to the participant's hand, through the recording of the SCR, corresponding to the activation of the autonomic nervous system (ANS) [26-28]. Since the response to a noxious stimulus starts before skin contact, as a consequence of anticipatory evaluation of the sensory consequence to the approaching stimulus [29], we also studied the modulation of such an anticipatory response to pain following FBI. We induced the FBI by manipulating the congruency of visuo-tactile stroking between the virtual body and participants' own body (stroking factor) and we manipulated whether the participants saw a virtual body or a control object on their head-mounted display (visual feedback configuration factor).

\section{Experiment 1}

\subsection{Materials and methods}

\subsubsection{Participants}

Fourteen right-handed healthy volunteers took part in Experiment 1 (mean age \pm standard deviation: $24.87 \pm 2.82$ years; 3 females). All participants had normal vision and were naive to the purpose of the experiment. All participants gave their written informed consent before the inclusion in the study. The study was approved by the local ethics committee,La Commission d'Ethique de la Recherche Clinique de la Faculté et de Medicine de l'Université de Lausanne,and was conducted in accordance with the ethical standards of the Declaration of Helsinki [30].

\subsubsection{Experimental setup}

The experiment was conducted in a light-shielded room where a robotic device for tactile stroking was installed [23]. The robotic device had $200 \mathrm{~cm} \times 90 \mathrm{~cm} \times 10 \mathrm{~cm}$ dimensions and a soft foam cover that permitted participants to lie comfortably on their back. Stroking units were integrated in the robotic device that allowed to separately stroke the left and right upper back of participants. A stroking unit consisted of an ultrasonic motor (Shinsei, USR60-E3 N, Japan, http://www.shinsei-motor.com) that actuated via a pinionhole mechanism movable end parts on which a spring blade and a plastic sphere were mounted. Plastic spheres reached through gaps in the foam cover of the robotic device to touch the upper back of a participant and via the spring blades adapted to the curvature of participants' back during stroking.

Visual stimuli were presented on a head-mounted display (Virtual Realities, Virtual Viewer 3D, Houston, Texas, www.vrealities.com/virtualviewer3d.html) with $800 \times 600$ pixel resolution and 35 degrees of visual angle. On headphones white noise was presented to participants in order to prevent them from hearing acoustic cues from the robotic stroking.

A serial keypad (Targus Numeric Keypad AKP10US, Anaheim, CA, www.targus.com) was used to record participants' button press responses, which were given with participant's right hand.

In-house software (ExpyVR, Lausanne, Switzerland, http://lnco.epfl.ch/expyvr) was used for visual and acoustic stimulus presentation and recording of responses and LABview software (National Instruments Corporation, version 2010b, Austin Texas, www.ni.com/labview) was used for robotic device control.

\subsubsection{Stimuli}

Tactile stroking by the robotic device was specified by pre-programmed stroking sequences. A total of four random sequences were created before the experiment with Matlab software (MathWorks, version R13, Massachusetts US, http://www.mathworks.ch). These sequences specified the position of a stroking unit at $100 \mathrm{~Hz}$ sampling rate, within $0-20 \mathrm{~cm}$ distance range, and $2-12 \mathrm{~cm} / \mathrm{s}$ velocity range. Within these limits, the four sequences had respectively random direction, timing, relative position, and speed.

The head-mounted display showed an image of a human body (male or female, according to participant's gender) wearing a white t-shirt and blue jeans against a gray background (virtual body, Fig. 1a) or a white rectangle, as a control condition (virtual object, Fig. 1b). The virtual body held a prone posture and was seen in bird's eye view [25].

\subsubsection{SCR device}

The BioSemi ActiveTwo system (ActiveTwo, BioSemi B.V., Amsterdam, Netherlands) was used as signal amplifier with specific GSR sensors consisting of 2 passive Nihon Kohden electrodes to induce an oscillator signal synchronized with the sample-rate. The sensors were applied on the distal phalanx of the index and middle finger of the left hand, while the two references electrodes were applied to the left forearm. A saline conductive paste was applied to the electrodes, in order to improve the signal-to-noise ratio.

Data were digitalized on a dedicated computer through optic connection with a sample rate of $2048 \mathrm{~Hz}$ and then data were resampled offline at $200 \mathrm{~Hz}$.

\subsubsection{Procedure}

An experimental run consisted of an FBI-induction phase, followed by a pain-stimulation phase, questionnaire ratings, and a resting period (Fig. 1d)

The FBI-induction phase consisted of 50 s visuo-tactile stroking in synchronous or asynchronous fashion (stroking factor) seen on an avatar or object (visual feedback factor).

A total of 8 trials were presented during the pain-stimulation phase. A trial began with visually presenting a needle that moved toward the body/object eventually contacting the target ("virtual puncture") during $5 \mathrm{~s}$ and was followed by a fixed interstimulus interval of $5 \mathrm{~s}$ after which the next trial was presented. During the pain-stimulation phase, visuo-tactile stroking was continuously presented. The picture of a big static needle was displayed 

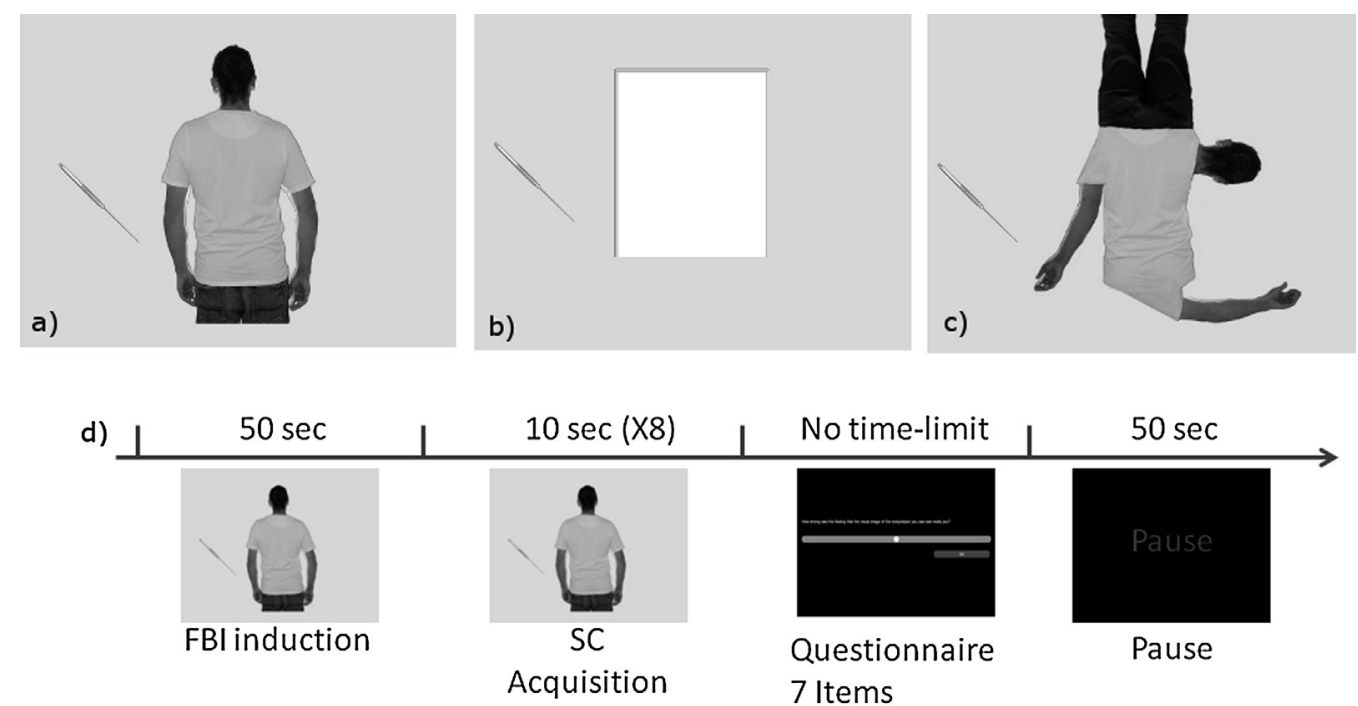

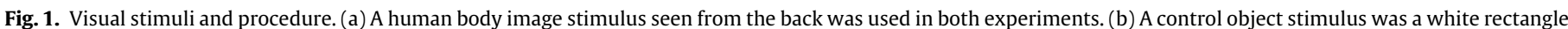

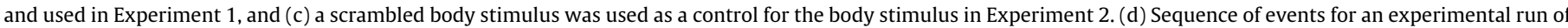

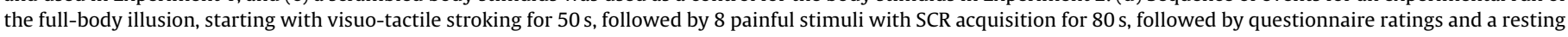
period of $50 \mathrm{~s}$.

on the left side of the virtual body/object during either the induction phase and the stimulation phase (Fig. 1). For half of the trials a "real contact" condition was presented. The biologic left hand of the participant was hit with a real needle synchronously with the contact of the virtual needle with the visual target. The real needle had a blunt end with $1 \mathrm{~mm}$ diameter and the contacting the skin was not invasive. The real contact stimulation was administered always by the same trained experimenter who was blind to the actual stroking condition. In the other half of trials, a "simulated contact" condition was presented. Participants did not receive any stimulation during the vision of the virtual pinprick. This condition was used to assess the presence of any SCR response to the vision of the noxious stimulus hitting the target in the absence of any somatosensory painful stimulation [27].

This procedure resulted in 8 different conditions: body congruent real, body congruent simulated, body incongruent real, body incongruent simulated, object congruent real, object congruent simulated, object incongruent real, and object incongruent simulated. After the pain-stimulation phase, the questionnaire was administered; $50 \mathrm{~s}$ of rest separated the different condition runs.

\subsubsection{Measures}

2.1.6.1. Questionnaire ratings. During each condition run and immediately after the pain-stimulation phase, participants were asked to complete a questionnaire comprising items adapted from previous studies on bodily illusions [4,9] and pain [20] (Table 1). Responses were given through a 7 points visual analogue scale (VAS) and were automatically coded by the experimental software with a score ranging from -3 to +3 .

Participants were asked to move the cursor along horizontal axes by pressing buttons with the index and ring fingers (left/right movement) with their right hand, while they confirmed their choice pressing the button in the center with the middle finger. The random sequences of either experimental conditions and questionnaire items were under computer control.

The data from each question underwent an intra-subject standardization by means of an ipsatization procedure in order to neutralize the effect in responses set [31,32]. Specifically, each rating was subtracted by the mean rating of the subject responses in all questions and conditions and then divided by the standard deviation of subject's responses in all questions and conditions.
2.1.6.2. Skin conductance response. The skin conductance level was recorded at DC level. An off-line digital high pass filter set at $0.05 \mathrm{~Hz}$ was applied to obtain phasic skin conductance responses [33]. This filter is effective to get back at level 0 the SCR after $1-3 \mathrm{~s}$ post-peak and highlights the event related responses in the skin conductance signal. The maximum amplitude of the SCR was used as measure of autonomous nervous system responses. For each trial, the maximum amplitude recorded in the time window of $7 \mathrm{~s}$ starting with the initial movement of the needle was extracted. The measures were intra-subject normalized [34-36] in order to obtain comparable measures among the participants, given the well known large inter-subject variability of SCR [37,38]. In addition, the mean skin conductance level (SCL) during each condition was calculated to evaluate the basal sympathetic tone [39].

\subsubsection{Data analysis}

Data were analyzed with STATISTICA 6.0 (StatSoft, Italy, http://www.statsoft.it).

A repeated measure ANOVA was run on SCR data in a $2 \times 2 \times 2$ within subject design. The main factors were: visual feedback configuration (body/object); stroking (congruent/incongruent) and

\section{Table 1}

Questionnaire items from Q1 to Q4 are questions inquiring about bodily illusory sensations and Q6 and Q7 are questions asking for explicit pain experience while Q5 is a control question. Questions sequence were fully randomized and under computer control.

\begin{tabular}{cl}
\hline & Question \\
\hline Q1 & $\begin{array}{l}\text { How strong was the feeling that the visual image of the } \\
\text { body/object you saw was really you? (self identification) }\end{array}$ \\
Q2 & $\begin{array}{l}\text { How strong was the feeling that you were drifting downwards or } \\
\text { upwards? } \\
\text { (self location). }\end{array}$ \\
Q3 & $\begin{array}{l}\text { How Strong was the feeling that you could control the movement } \\
\text { of the body you saw? (agency over the visual object) }\end{array}$ \\
Q4 & $\begin{array}{l}\text { How strong was the feeling that you cannot move your own body? } \\
\text { (loss of agency) }\end{array}$ \\
Q5 & $\begin{array}{l}\text { How strong was the feeling that you had more than two bodies? } \\
\text { (control question) }\end{array}$ \\
How much intense was the pain inflicted by the needle? \\
(pain intensity)
\end{tabular}


stimulus contact (real/simulated). When a significant effect was found, the $\eta^{2}$ effect size and power were computed.

Ipsatization transformed questionnaire ratings in Z-scores with a normal distribution allowing a proper use of parametric tests on questionnaire data $[31,32]$. Separated repeated measures ANOVAs were conducted for each different question on ipsatized values and on SCL measures. Each one of these ANOVAs resulted in a $2 \times 2$ within subject design factoring visual feedback configuration (body/object) and stroking (congruent/incongruent).

Significant level was set at $p<.05$, when a significant interaction was detected; post-hoc analysis were conducted with Fisher LSD test.

In addition, the Pearson's $r$ correlation was calculated between questionnaire responses and SCR.

\subsection{Results}

\subsubsection{Skin conductance}

The ANOVA on SCR data showed a main effect of contact $\left(F(1,13)=20.589 ; p<.001, \eta^{2}=.613\right.$; power $\left.=.987\right)$ and an interaction between visual feedback and stroking $(F(1,13)=6.111 ; p<.05$; $\eta^{2}=.320$; power $=.942$ ). The other main factors (visual feedback: $F(1,13)=2.221 ; p=.16$; stroking: $F(1,13)=.561 ; p=.47)$ and interactions were not significant (visual feedback $\times$ contact: $F(1,13)=.232$; $p=.64$; congruency $\times$ contact: $F(1,13)=4.133 ; p=.063$; visual feedbackcongruency $\times$ contact: $F(1,13)=.084 ; p=.77)$. Fisher's post-hoc tests showed that the real contact $(Z=.3 \pm .08)$ induced a greater SCR than the simulated contact $(Z=-.4 \pm .08)$. The interaction between visual feedback and stroking notably showed that, during the visual feedback of the body, the SCR was lower for congruent versus incongruent stroking while for the object visual feedback the trend was in the opposite direction. Post-hoc testing revealed that the body congruent condition $(Z=-.28 \pm .05)$ had different responses from body incongruent $(Z=.03 \pm .09 ; p=.026)$ and object congruent conditions $(Z=.08 \pm .13 ; p=.013)$. At the same time the other three conditions did not show statistically significant differences in any direct comparison (Fig. 2a)

The ANOVA on SCL did not show any significant effect nor for the main effects (visual feedback: $F(1,13)=1.288$; $p=.277$; stroking: $F(1,13)=3.049 ; p=.104)$, neither for the interaction $(F(1,13)=1.765 ; p=.207)$.

\subsubsection{Questionnaire}

The analysis of self-identification ratings (Q1) showed the main effect of visual feedback $\left(F(1,13)=15.81 ; p \leq .001, \eta^{2}=.549\right.$; power $=.956$ ) but no main effect of stroking and no visual feedback $x$ stroking interaction. Thus, self-identification was rated significantly higher in the body $(Z=.91 \pm .26)$ conditions than in the object conditions $(Z=-.22 \pm .23)$.

The ANOVA for self-location ratings (Q2) showed a visual feedback $x$ stroking interaction $\left(F(1,13)=5.29 ; p<.05 ; \eta^{2}=.29\right.$; power $=.904$ ). Post hoc comparisons showed that values were lower in the object incongruent condition $(Z=-1.79 \pm .39)$ than in the other three conditions (body congruent $=-1.28 \pm .54$; body incongruent $=-1.28 \pm .49 ;$ object congruent $=-.86 \pm .51$ ), which were at the same level (all $p \leq .05$ ). There was no main effect of visual feedback and no main effect of stroking for self-location ratings (Q2).

Questions about agency (Q3 and Q4), the control question (Q5), and questions about pain experience (Q6 and Q7) revealed no significant main effects and no interactions. The absence of significant differences for the questions about pain experience (Q6 and Q7) suggests that our experimental manipulation did not result in consciously reportable effects on pain experience (Fig. 2b).
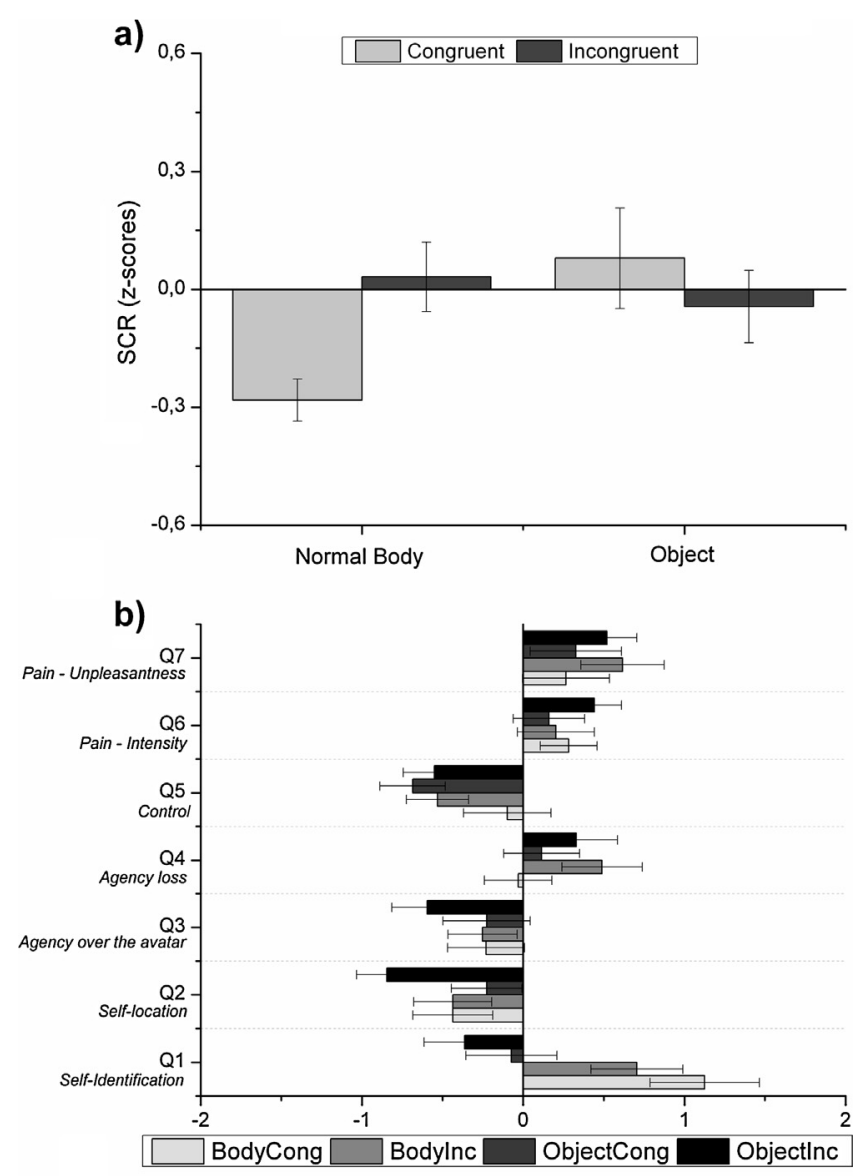

Fig. 2. Experiment 1 results. (a) Columns represent the mean SCR response for the significant interaction between visual feedback (body/object) and stroking (congruent/incongruent). The SCR has been transformed in z-scores. The panel (b) shows mean questionnaire ratings. The thin bars indicate standard errors.

\subsubsection{Correlation analysis}

Correlation analysis showed a significant negative correlation between self-identification ratings (Q1) and real contact SCR $(r=-.27 ; p<.05)$, that is, a high degree of self-identification was associated with low SCR. None of the other questions showed significant correlations with the implicit measures.

\section{Experiment 2}

\subsection{Materials and methods}

\subsubsection{Participants}

Sixteen right-handed healthy volunteers participated (Age \pm SD: $23.56 \pm 2.50$ years, 4 females), who had not participated in Experiment 1 and were thus naïve to the purpose of the experiment. All participants had normal vision and gave their written informed consent before the inclusion in the study. The study was approved by the local ethics committee, La Commission d'Ethique de la Recherche Clinique de la Faculté et de Medicine de l'Université de Lausanne, and was conducted in accordance with the ethical standards of the Declaration of Helsinki [30].

\subsubsection{Materials, methods, and procedures}

The materials, methods, procedures, and analysis were the same as in Experiment 1, except for the following differences.

In Experiment 2, we investigated whether any visual effect of self-identification on SCR needed the visual observation of a realistic and anatomically intact body. For this purpose, we 
presented participants with either a virtual human body (similar to Experiment 1), or with a scrambled version of the same body, shaped with anatomically impossible limb configuration (Fig. 1c). More precisely, the trunk of the virtual body was presented at the center of the image and similarly to the original image, and the other body segments were presented at incongruent positions (Fig. 1c). The scrambled body image was created with GIMP software (GIMP 2.6.10; www.gimp.org) and was a modification of the avatar image in such a way that we provided a unitary picture that could be processed also as a whole and not just as a fragmented summation of smaller figures. We named this condition "scrambled body". The experimental design resulted in a $2 \times 2 \times 2$ within subjects design factoring: visual body configuration (anatomical/scrambled body), stroking (congruent/incongruent) and stimulus contact (real/simulated) for the SCR analysis, and a series of $2 \times 2$ within subjects design factoring: visual body configuration (anatomical/scrambled body), stroking (congruent/incongruent) for questionnaire and SCL analysis.

\subsection{Results}

\subsubsection{Skin conductance}

The ANOVA on SCR data showed a main effect of contact $\left(F(1,15)=68.148 ; p<.001, \eta^{2}=.819\right.$; power $\left.>.999\right)$ and a main effect of visual feedback $\left(F(1,15)=34.909, p<.001 ; \eta^{2}=.699\right.$ power $\left.>.999\right)$ moreover the interaction between visual feedback and stroking factors was significant $\left(F(1,15)=6.46 ; p<.05 ; \eta^{2}=.301\right.$; power $\left.=.95\right)$ as well as the interaction between visual feedback and contact $\left(F(1,15)=11.221 ; p<.01, \eta^{2}=.428\right.$; power $\left.=.997\right)$. The main factor stroking $(F(1,15)=.417 ; p=.528)$ and the other interactions were not significant (stroking $\times$ contact: $F(1,15)=.056 ; p=.817$; visual feedback $\times$ stroking $\times$ contact: $(F(1,15)=.024 ; p=.879)$.

The real contact $(Z=.37 \pm .04)$ induced a greater SCR than the simulated contact $(Z=-.38 \pm .04)$. The visual feedback main effect showed that independently from the stroking main effect, seeing a virtual body in anatomical configuration $(Z=-.19 \pm .03)$ induced lower SCR to painful stimuli than seeing a scrambled body $(Z=.18 \pm .03)$. The interaction between visual feedback and stroking factors, congruently with Experiment 1 and our prediction, showed that, using the anatomical configuration as a visual feedback, the SCR was lower for congruent than for incongruent stroking while for the scrambled body there was an opposite trend. Post hoc comparisons showed that the body congruent condition $(Z=-.33 \pm .05)$ differed significantly from scrambled congruent condition $(Z=.31 \pm .09 ; p<.001)$ and scrambled incongruent $(Z=.05 \pm .09 ; p<.05)$, moreover a difference close to significance was found between anatomical body congruent and anatomical body incongruent $(Z=-.05 \pm .06 ; p=.08)$ contrast (Fig. 3a).

The post hoc analysis for the visual body configuration by contact interaction showed that the main effect of visual body configuration was driven by differences in the real contact conditions as the real touch during anatomical body conditions $(Z=.06 \pm .07)$ differed significantly from the real touch during scrambled body configurations $(Z=.68 \pm .08 ; p<.001)$. Moreover, both were stronger than the simulated contact conditions (all $p<.001$ ), which did not show significant difference for the two visual body configurations (simulated anatomical body $=-.45 \pm .05$, simulated scrambled body $=-.31 \pm .06 ; p=.178$ ).

Consistently with Experiment 1, the ANOVA on SCL did not show significant results nor for the main effects (visual feedback: $F(1,15)=.647 ; p=.434$; stroking: $F(1,15)=.067 ; p=.798)$, neither for the interaction $(F(1,15)=.096 ; p=.761)$.

\subsubsection{Questionnaire data}

The ANOVA for self-identification ratings (Q1) showed significant main effects of visual body configuration $(F(1,13)=5.99$;
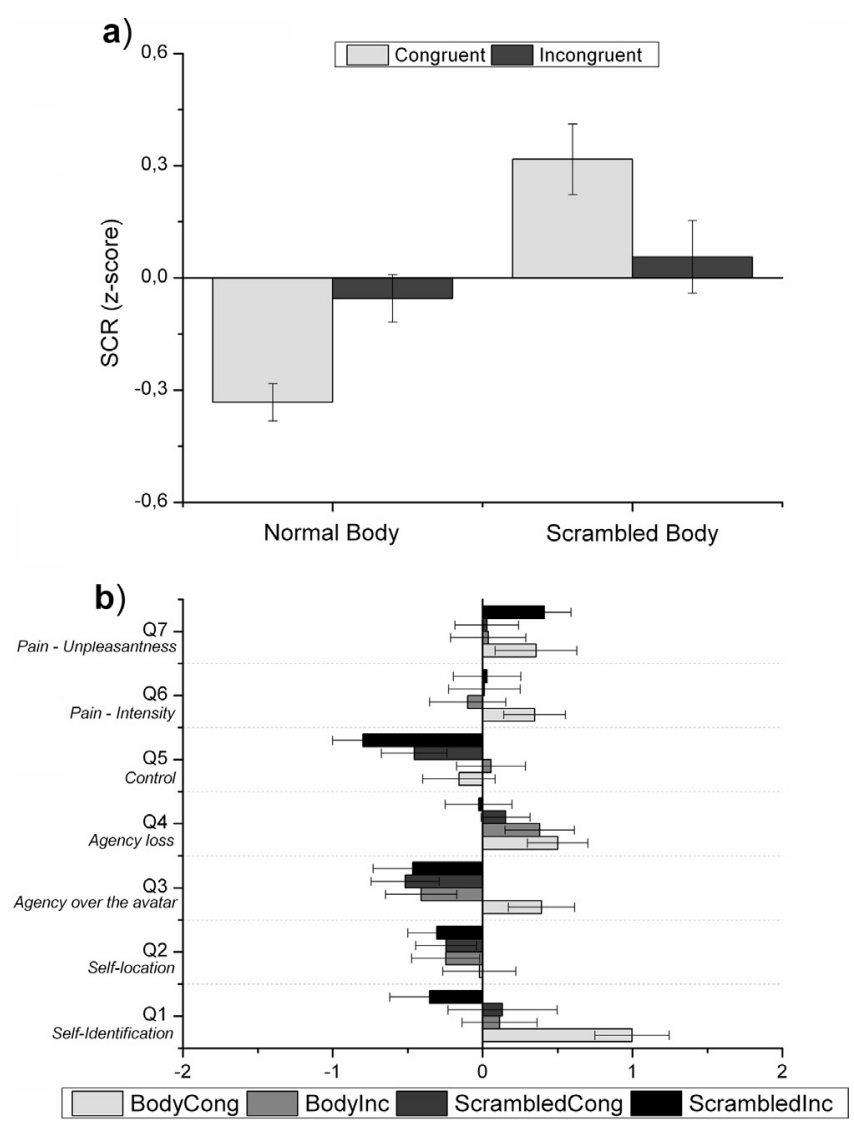

Fig. 3. Experiment 2 results. (a) Columns represent the mean SCR response for the significant interaction between visual feedback (body/object) and stroking (congruent/incongruent). The SCR has been transformed in z-scores. The panel (b) shows mean questionnaire ratings. The thin bars indicate standard errors.

$p<.05 ; \eta^{2}=.285$; power $\left.=.629\right)$ and stroking $(F(1,13)=5.04 ; p<.05$; $\eta^{2}=.251$; power $\left.=.556\right)$. There was no visual body configuration by stroking interaction. The anatomical body $(Z=.55 \pm .18)$ conditions induced higher self-identification ratings than the scrambled body conditions $(Z=-.11 \pm .26)$. Moreover, the congruent stroking $(Z=.56 \pm .24)$ induced higher ratings compared to the incongruent $(Z=-.12 \pm .22)$ suggesting an additive but not interactive effect which identified the anatomical body congruent stroking condition as the one with the stronger effect of self-identification with the human picture.

Questions about self-location (Q2) and agency (Q3 and Q4) did not reveal any significant main effect and no interaction.

The control question (Q5) showed a significant main effect of the visual feedback $\left(F(1,13)=10.23 ; p<.01 ; \eta^{2}=.406\right.$; power $=.997$; body $=-.05 \pm .19$; scrambled $=-.63 \pm .18$ ). Consistent with Experiment 1, none of the questions about pain experience (Q6 and Q7) showed significant main effects or interactions.

\subsubsection{Correlation}

Correlation analysis showed a significant negative correlation between self-identification ratings (Q1) and real contact SCR $(r=-.31 ; p<.05)$. No other questionnaire items showed a significant correlation with the SCR.

\section{Discussion}

In the present study, we found that changes in self-identification with a virtual body modulate arousal responses to acute painful stimulations, as reflected by a decreased SCR. Although, external stimuli that evoke nociceptive afferent signals are the main 
contributor to pain experience, other internal factors contribute to the genesis and the modulation of the pain experience, such as generically defined cognitive or affective components. Among these factors, it has been possible to differentiate affectivemotivational components, such as emotions or meditation-induced states [40,34], from more cognitive factors, such as attention and expectations related to incoming stimuli features [41,42,29]. Moreover, perceptual factors, such as proprioception [43] and the visual size of a body part play a role [44].

Looking at one's own body but not looking at a neutral object or at another person's body has been reported to induce analgesia for acute painful stimulation $[22,20]$. Here, we sought for a similar modulation of pain responses induced by the vision of a virtual body that also depended upon the level of illusory self-identification with that body during a full-body illusion [9] induced by controlled robotic stimulations [23-25].

In the first experiment, we compared automatic responses to acute painful stimulations, while a virtual body was shown in back-view on an HMD. These responses were compared with those obtained when seeing a control neutral object $[26,45,46,9,8]$. Our results revealed that the SCR to painful stimuli decreased for real and simulated noxious stimuli, specifically under congruent visuotactile stimulation and when the body, but not the object, was seen.

This result was further qualified by the second experiment, where another control condition was added, consisting of a scrambled human figure, typically used in studies testing body and face perception [47-49]. The specific aim of Experiment 2 was to assess the importance of a realistic body configuration [48] for inducing the FBI and the reduction in the SCR to painful stimuli. The arousal responses to the applied pinpricks, both real and simulated, showed, consistently with Experiment 1, a selectively decreased SCR, only when the body was shown in the anatomical configuration and under congruent visuo-tactile stimulation. Moreover, negative results from SCL analysis suggested that the changes in arousal responses that we observed were related more to the transient event related response than to a modification of an altered basal sympathetic tone [39].

The present results add to previous findings on the modulation of pain experience by vision. Vision of noxious stimuli seen as approaching another person's body induces arousal responses in healthy humans [27]. It has been argued that such responses are mainly based on the cognitive evaluation of the approaching stimulus which would produce an automatic anticipatory response $[29,50]$ that has been hypothesized to be mediated by emphatic sharing of the affective component of pain [51,52]. Furthermore, previous works reported that the vision of one's own stimulated body parts can modulate pain thresholds $[20,44]$ or the rating of pain intensity [22], suggesting an analgesic effect when looking at one's own body. The present study goes beyond such previous results by linking the visual response to an incoming threat directed towards another person's body, to illusory self-identification experienced towards an observed virtual body.

We hypothesized that the increased self-identification, when seeing the virtual body stroked in a congruent fashion, would be reflected in changes in the processing of painful stimuli akin to those described during the direct observation of one's own body typically consisting of a reduced response to painful stimuli $[22,20,44]$. Questionnaire data showed that higher selfidentification was recorded with the avatar only when presented with an anatomically correct body configuration and when stroked congruently, according to the literature $[4,53,28,9,8]$.

Investigating the relationship between the self-identification and reduced SCR further, we found that the overall correlation between the magnitude of illusory self-identification and the magnitude of SCR was significant. The correlation was negative in both experiments, sustaining that the more self-identification with the avatar our participants reported, the stronger was the reduction of the SCR for painful stimuli. Although, in the correlation analysis all conditions were considered together with the potential risk of an autocorrelation bias, it is noteworthy that illusory selfidentification was the only item from a total of 7 items that showed the same negative correlation with SCR in experiments 1 and 2.

Our findings of a reduced arousal response are in line with earlier studies reporting elevated pain thresholds when seeing a body part or when self-identifying with a virtual body $[45,20]$, but also extend these data on visual analgesia described earlier. In particular, we show here that such decreased arousal response to painful stimuli is tuned by bodily self-consciousness and also modulates anticipatory levels of painful stimuli processing and thus is not strictly dependant on the somatosensory nociceptive input. However whereas these pain thresholds data have recently been extended with explicit ratings of pain experience [22], we did not observe similar effects in explicit pain ratings in experiment 1 and 2. Consistently with our results, a recent study (conducted in two different laboratories) showed that explicit pain ratings for stimuli delivered to the biological hand did not change during the RHI [54]. It is worth noting that we assessed pain ratings only once for each condition during the questionnaire phase, and not on a trial by trial basis after each stimulation, in order to reduce possible interference with the induced illusory state and skin conductance recording. However, this procedure of measuring pain ratings only once might weaken the confidence with pain ratings as several external confounding effects, like memory or other post-perceptual processes, might have interfered with the judgment of stimulations which was delayed.

It has also been proposed that the body is processed as a whole, as suggested by the reported advantage for a global processing of body pictures shown in an upright posture as compared to upsidedown or non-anatomical postures $[55,48]$. In the current study, we showed that in order to induce the FBI, the picture of the avatar needs to be presented in its correct anatomical configuration. Although, modulation of pain experience for an isolated body part was found when looking at the body part [20,44], the present data, comparing the observed effects for the full normal versus scrambled body, suggest that global bodily processing of a seen human body impacts self-identification and arousal responses to painful stimuli. Interestingly, although we presented avatars that matched the gender of our participants, the avatars appearances were different with respect to the specific hair style, or the skin colour of participants. However, our results indicate that primarily the anatomical configuration of the avatar defined the possibility to increase the self-identification with it, and not its actual similarity with participant's body, congruently with the finding that even an opposite gender avatar could induce embodiment effects [56,57].

In conclusion, we found that it is possible to reduce the implicit arousal response to acute painful stimuli throughout the full body illusion. This effect is already available during the anticipatory response to the incoming expected painful stimulation; it is related to the degree of self-identification with the stroked picture and would be achieved only for pictures of human bodies presented with a normal anatomical configuration. However, this implicit arousal reduced response is not transferred in an aware reduced experience of pain. Our data suggests that pain processing shares functional mechanism with self-identification and extends previous research towards a fundamental understanding of selfconsciousness.

\section{References}

[1] Blanke O, Metzinger T. Full-body illusions and minimal phenomenal selfhood. Trends Cogn Sci 2009;13(1):7-13. 
[2] Blanke O. Multisensory brain mechanisms of bodily self-consciousness. Nat Rev Neurosci 2012:556-71.

[3] Ferri F, Frassinetti F, Ardizzi M, Costantini M, Gallese V. A sensorimotor network for the bodily self. J Cogn Neurosci 2012:1584-95.

[4] Botvinick M, Cohen J. Rubber hands feel touch that eyes see. Nature 1998;391(6669):756.

[5] Costantini M, Haggard P. The rubber hand illusion: sensitivity and reference frame for body ownership. Conscious Cogn 2007;16(2):229-40.

[6] Lloyd DM. Spatial limits on referred touch to an alien limb may reflect boundaries of visuo-tactile peripersonal space surrounding the hand. Brain Cogn 2007;64(1):104-9.

[7] Perez-Marcos D, Slater M, Sanchez-Vives MV. Inducing a virtual hand ownership illusion through a brain-computer interface. NeuroReport 2009;20(6):589-94.

[8] Tsakiris M, Carpenter L, James D, Fotopoulou A. Hands only illusion: multisensory integration elicits sense of ownership for body parts but not for non-corporeal objects? Exp Brain Res 2010;204(3):343-52.

[9] Lenggenhager B, Tadi T, Metzinger T, Blanke O. Video ergo sum: manipulating bodily self-consciousness. Science 2007;317(5841):1095-9.

[10] van der Hoort B, Guterstam A, Ehrsson HH. Being Barbie: the size of one's own body determines the perceived size of the world. PloS one 2011;6(5):e20195.

[11] Tsakiris M. My body in the brain: a neurocognitive model of body-ownership. Neuropsychologia 2010;48(3):703-12.

[12] Aspell JE, Lenggenhager B, Blanke O. Keeping in touch with one's self: multisensory mechanisms of self-consciousness. PloS one 2009;4(8):e6488.

[13] Ehrsson HH. The experimental induction of out-of-body experiences. Science 2007;317(5841):1048.

[14] Petkova VI, Björnsdotter M, Gentile G, Jonsson T, Li T-Q, Ehrsson HH. From part- to whole-body ownership in the multisensory brain. Current Biol 2011;21(13):1118-22.

[15] Ehrsson HH, Holmes NP, Passingham RE. Touching a rubber hand: feeling of body ownership is associated with activity in multisensory brain areas. J Neurosci 2005;25(45):10564-73.

[16] Salomon R, van Elk M, Aspell JE, Blanke O. I feel who I see: visual body identity affects visual-tactile integration in peripersonal space. Conscious Cogn 2012;21(3):1355-64.

[17] Tsakiris M, Hesse MD, Boy C, Haggard P, Fink GR. Neural signatures of body ownership: a sensory network for bodily self-consciousness. Cerebral Cortex 2007;17(10):2235-44.

[18] Haggard P, Iannetti GD, Longo MR. Spatial sensory organization and body representation in pain perception. Current Biol 2013;23(4):R164-76.

[19] Iannetti GD, Mouraux A. From the neuromatrix to the pain matrix (and back). Exp Brain Res 2010;205:1-12.

[20] Longo MR, Betti V, Aglioti SM, Haggard P. Visually induced analgesia: seeing the body reduces pain. J Neurosci 2009;29(39):12125-30.

[21] Lenz FA, Casey KL, Jones EG, Willis WD. Organization of the central pain pathways. In: The human pain system. Cambridge: Cambridge University Press; 2010. p. 64-195.

[22] Longo MR, Iannetti GD, Mancini F, Driver J, Haggard P. Linking pain and the body: neural correlates of visually induced analgesia. J Neurosci 2012;32(8):2601-7.

[23] Duenas J, Chapuis D, Pfeiffer C, Martuzzi R, Ionta S, Blanke O, Gassert R. Neuroscience robotics to investigate multisensory integration and bodily awareness. IEEE Eng Med Biol Soc Conf 2011;2011:8348-52.

[24] Ionta S, Heydrich L, Lenggenhager B, Mouthon M, Fornari E, Chapuis D, et al. Multisensory mechanisms in temporo-parietal cortex support self-location and first-person perspective. Neuron 2011;70(2):363-74.

[25] Pfeiffer C, Lopez C, Schmutz V, Duena JA, Martuzzi R, Blanke O. Multisensory origin of the subjective first-person perspective: visual, tactile, and vestibular mechanisms. PloS One 2013;8(4):e61751.

[26] Armel KC, Ramachandran VS. Projecting sensations to external objects: evidence from skin conductance response. Proc R Soc B: Biol Sci 2003;270(1523):1499-506.

[27] Forgiarini M, Gallucci M, Maravita A. Racism and the empathy for pain on our skin. Front Psychol 2011;2:108.

[28] Guterstam A, Petkova VI, Ehrsson HH. The illusion of owning a third arm. PloS One 2011;6(2):e17208.

[29] Clark JA, Brown CA, Jones AKP, El-Deredy W. Dissociating nociceptive modulation by the duration of pain anticipation from unpredictability in the timing of pain. Clin Neurophysiol 2008;119(12):2870-8.
[30] World Medical Organization. Declaration of Helsinki. $\mathrm{Br}$ Med J 1996;313(7070):1448-9.

[31] Broughton R, Wasel N. A text-stimuli presentation manager for the IBM PC with ipsatization correction for response sets and reaction times. Behav Res Meth Ins C 1990;22(4):421-3.

[32] Cattell RB. Psychologicalmeasurement: ipsative, normative and interactive. Psychol Rev 1944;51:292-303.

[33] Andreassi JL. Psychophysiology: human behavior \& physiological response. 5th edn. Mahwah: Lawrence Erlbaum Associates; 2000.

[34] Rhudy JL, Bartley EJ, Williams AE. Habituation, sensitization, and emotional valence modulation of pain responses. Pain 2010;148(2):320-7.

[35] Rhudy JL, Williams AE, McCabe KM, Russell JL, Maynard LJ. Emotional control of nociceptive reactions (ECON): do affective valence and arousal play a role? Pain 2008;136(3):250-61.

[36] Williams AE, Rhudy JL. Emotional modulation of autonomic responses to painful trigeminal stimulation. Int J Psychophysiol 2009;71(3):242-7.

[37] Fowles DC, Christie MJ, Edelberg R, Grings WW, Lykken DT, Venables PH. Publication recomandations for electrodermal measurements. Psychophysiology 1981;18(3):232-9.

[38] Lykken DT, Venables PH. Direct measurement of skin conductance: a proposal for standardization. Psychophysiology 1971;8(5):656-72.

[39] Nagai Y, Critchley HD, Featherstone E, Trimble MR, Dolan RJ. Activity in ventromedial prefrontal cortex covaries with sympathetic skin conductance level: a physiological account of a default mode of brain function. NeuroImage 2004;22(1):243-51.

[40] Brown CA, Jones AKP. Meditation experience predicts less negative appraisal of pain: electrophysiological evidence for the involvement of anticipatory neural responses. Pain 2010;150(3):428-38.

[41] Brown CA, Jones AKP. A role for midcingulate cortex in the interruptive effects of pain anticipation on attention. Clin Neurophysiol 2008;119(10): 2370-9.

[42] Brown CA, Seymour B, Boyle Y, El-Deredy W, Jones AKP. Modulation of pain ratings by expectation and uncertainty: behavioral characteristics and anticipatory neural correlates. Pain 2008;135(3):240-50.

[43] Gallace A, Torta DME, Moseley GL, Iannetti GD. The analgesic effect of crossing the arms. Pain 2011;152(6):1418-23.

[44] Mancini F, Longo MR, Kammers MPM, Haggard P. Visual distortion of body size modulates pain perception. Psychol Sci 2011;22:325-30.

[45] Hänsel A, Lenggenhager B, von Känel R, Curatolo M, Blanke O. Seeing and identifying with a virtual body decreases pain perception. Eur J Pain 2011;15: 874-9.

[46] Hohwy J, Paton B. Explaining away the body: experiences of supernaturally caused touch and touch on non-hand objects within the rubber hand illusion. PloS one 2010;5(2):e9416.

[47] Hershler O, Hochstein S. At first sight: a high-level pop out effect for faces. Vision Res 2005;45(13):1707-24

[48] Reed CL, Stone VE, Bozova S, Tanaka J. The body-inversion effect. Psychol Sci $2003 ; 14(4): 302-8$.

[49] Reed CL, Stone VE, Grubb JD, McGoldrick JE. Turning configural processing upside down: part and whole body postures. J Exp Psychol: Hum Percept Perform 2006;32(1):73-87.

[50] Ploghaus A. Dissociating pain from its anticipation in the human brain. Science 1999;284(5422):1979-81.

[51] Hein G, Singer T. I feel how you feel but not always: the empathic brain and its modulation. Curr Opin Neurobiol 2008;18(2):153-8.

[52] Singer T, Seymour B, O’Doherty J, Kaube H, Dolan RJ, Frith CD. Empathy for pain involves the affective but not sensory components of pain. Science 2004;303(5661):1157-62.

[53] Ehrsson HH, Spence C, Passingham RE. That's my hand! Activity in premotor cortex reflects feeling of ownership of a limb. Science 2004;305:875-7.

[54] Mohan R, Jensen KB, Petkova VI, Dey A, Barnsley N, Ingvar M, McAuley JH, Moseley LG, Ehrsson HH. No pain relief with the rubber hand illusion. PloS One 2012;7(12):e52400.

[55] Bosbach S, Knoblich G, Reed CL, Cole J, Prinz W. Body inversion effect without body sense: insights from deafferentation. Neuropsychologia 2006;44(14):2950-8.

[56] Petkova VI, Ehrsson HH. If I were you: perceptual illusion of body swapping. PloS One 2008;3(12):e3832.

[57] Slater M, Spanlang B, Sanchez-Vives MV, Blanke O. First person experience of body transfer in virtual reality. PloS One 2010;5(5):e10564. 